\title{
La práctica y la profesión del artista en el Islam: arquitectos y constructores en el al-Andalus omeya
}

\author{
JUAN A. SOUTO*
}

RESUMEN

Se trata de la práctica y la profesión del artista en el Islam. El límite espacio-temporal es al-Andalus bajo el dominio efectivo de la dinastía Omeya (711-1013). La actividad estudiada es la construcción.

\section{ABSTRACT}

This paper deals with the practice and profession of the artist in Islam. The chronological and geographical limits of our scope is al-Andalus under the effective dominion of the Umayyad dynasty (711-1013 A. D.); building is the studied activity.

\section{INTRODUCCIÓN}

Tratar de "la práctica y la profesión del artista» en el Islam es difícil y complejo. En primer lugar, debemos definir el propio campo en que se sitúa nuestro objeto de estudio, en este caso el Islam: desde un punto de vista histórico, se trata de la última de las religiones abrahámicas, cuyos otros dos exponentes son el Judaísmo y el Cristianismo, entendido el término "religión" como "forma de vida y comportamiento cuya doctrina emana de unas fuentes concretas". En este caso, del Corán, libro donde se contiene la palabra de Dios, y de la zuna, conjunto de tradiciones relativas a Muhammad, último Profeta del ciclo abrahámico. El Islam, expandido mediante conquista a lo largo de los siglos VII y vIII de la era cris-

\footnotetext{
* Departamento de Estudios Árabes e Islámicos. Universidad Complutense de Madrid.
} 
tiana, llegó a abarcar todo un amplio abanico de países, gentes y lenguas. Es por ello que desde un punto de vista geográfico llamamos "Islam» al conjunto de las naciones o pueblos que siguen esa religión cuya doctrina se encuentra contenida en el Corán y las enseñanzas de Muhammad. Así, usamos el término «lslam» como sinónimo de «Mundo islámico».

La extensión cronológica del Islam comprende desde el primer cuarto del siglo VII hasta nuestros días. La territorial, desde Filipinas a Marruecos -en su día, a la Península lbérica-y desde los Balcanes al África tropical. Vemos que son unos límites espacio-temporales realmente enormes, dentro de los cuales caben unas diferencias no menos amplias tanto de paisajes como de tipos humanos y hechos históricos políticos, sociales, culturales, artísticos...-. Pretender, pues, hablar de "la práctica y la profesión del artista" en el Islam a lo largo de unas pocas páginas sería un ejercicio tan pretencioso como inútil: ¿Por dónde empezar?, ¿por los orfebres de la Arabia del Profeta? ¿Dónde terminar?, ¿en los actuales tintoreros indonesios? ¿Cuánto espacio dedicar a cada aspecto? Es evidente que se impone un marco reducido, a modo de «muestra» o «ejemplo».

Como tal marco hemos elegido un espacio: al-Andalus, la Península Ibérica bajo dominio político islámico; un lapso de tiempo: el período de gobierno efectivo en ella de la dinastía Omeya (711-1013); y una actividad determinada: la construcción. Quien lea observará que tampoco es demasiado lo que se puede decir al respecto, ya que hay tres factores que dificultan el estudio de este tema, por concreto que parezca. Esos factores son la escasez de las fuentes con que contamos, su dispersión y la parquedad de sus datos. A ello hay que sumar la relativa falta de estudios sistemáticos de que han sido objeto esas fuentes en relación con el asunto de nuestro interés. La historia de la construcción y de los constructores en al-Andalus está por escribirse.

Al igual que en otros trabajos nuestros dirigidos a un público no necesariamente especializado, en el presente hemos prescindido de notas a pie de página, a fin de aligerar un escrito denso y de lectura que reconocemos difícil. Por el mismo motivo, y por razones de comodidad tipográfica, hemos prescindido de signos diacríticos al transliterar palabras árabes. Al final incluimos una lista de las fuentes citadas, con indicación de sus ediciones y traducciones, y bibliografía básica comentada, de manera que quien lo desee pueda saber de dónde proceden nuestros datos y profundizar en ellos o ampliarlos. También hemos añadido un vocabulario con todos los términos árabes y con los arabismos castellanos infrecuentes 
que aparecen a lo largo del trabajo. En último lugar se encontrará una lista de los emires y califas Omeyas andalusíes.

\section{LAS FUENTES DISPONIBLES: SU TIPOLOGIA}

Las fuentes para el estudio de la construcción en al-Andalus omeya son de varios tipos: literarias, geográficas, históricas, epigráficas, gliptográficas, biográficas, jurídicas y arqueológicas, fundamentalmente. Se comprenderá que datos de unas y de otras se imbriquen con frecuencia, asi como las metodologías de trabajo a aplicar. Recordemos entonces la necesidad, para obtener resultados satisfactorios, de realizar enfoques plurisciplinares.

En primer lugar, debemos plantearnos cómo son y qué clases de datos pueden aportarnos las fuentes relacionadas, pues estas poseen gran variedad tipológica y gran disparidad de objetivos, y sus autores se encuentran muy distanciados en el tiempo y en ocasiones muy alejados espacialmente entre sí. De todo lo cual se deriva una consecuencia bien condensada en frase de Labarta y Barceló: "Cuanto más tardía es una fuente, más precisos son los datos, más abundantes los calificativos, más detalladas las descripciones y más lejana la realidad".

Las fuentes literarias, tanto en prosa como en verso, hacen a veces referencia a construciones y constructores. No es ese, por supuesto, su objetivo más frecuente, ni suelen cumplirlo con precisión suficiente como para que podamos contar con ellas a manera de canteras de datos fiables. En gran medida recogen hechos exagerados o simplemente ficticios o legendarios.

Las fuentes geográficas, que a su vez comprenden varios sub-tipos, son de carácter fundamentalmente descriptivo. Su utilidad para el estudio de la construcción y de los constructores radica en que con relativa frecuencia dejan constancia de obras hechas en cierto lugar y por orden o bajo el patrocinio o mecenazgo de determinado personaje. A veces describen edificios, algunos de sus elementos y de sus materiales y hasta la procedencia de estos últimos. Con suerte, otro tanto ocurre respecto de los protagonistas directos de su erección. Sin embargo, y salvo excepciones, la imprecisión y la vaguedad son las notas dominantes en el terreno que nos ocupa.

De todas las fuentes escritas, las históricas son - junto con las epigráficas - las que más datos aportan acerca del tema de nuestro inte- 
rés. Por su propia naturaleza, y en muchos casos por sus propios objetivos particulares - propaganda más o menos encubierta del poder que hace las veces de mecenas-, intentan resaltar los hechos más notables de los soberanos y sus agentes. $Y$ entre tales hechos las construcciones ocupan un lugar destacado: recuérdese la relación cerrada, en todos tiempo y lugar, entre construcción y escenificación del poder y de quien lo encarna. En este sentido son más abundantes en noticias y más detallistas que las geográficas, pero con todo suelen carecer de datos precisos acerca de los constructores en sí, salvo destacadas excepciones.

Las fuentes epigráficas son el complemento fundamental de las geográficas y las históricas. Frente a los datos transmitidos por estas, siempre seleccionados, a menudo manipulados de diversas maneras y de una u otra forma sometidos al "paso" de unos a otros copistas, las inscripciones son documentos "directos» y "de primera mano". Por lo demás, si en las fuentes geográficas e históricas «hablan los escritores" -o sus mecenas a través de ellos-, la epigrafía permite en cierto modo «hablar a los edificios». Las inscripciones constructivas recogen a menudo las fechas precisas de realización de las obras conmemoradas y los nombres, cargos y oficios de sus artífices, aunque hoy día no tengamos demasiado claro cuáles eran los papeles exactos de estos.

Estrechamente ralacionada con la epigrafía está la gliptografía, ciencia que estudia las marcas de cantero y los grafitos, fuentes directísimas, más aún que la epigrafía "pura", al fin y al cabo fruto en muchas ocasiones de redacciones de cancilleria o de taller oficial.

Las fuentes biográficas, como su nombre indica, son repertorios de personajes con relación de sus vidas y sus hechos memorables. Los datos sobre construcciones y constructores son escasos y casi siempre colaterales y circunstanciales respecto de aquellos que interesa resaltar.

Las fuentes jurídicas no han sido muy exploradas desde el punto de vista de la historia de la construcción y de los constructores, por lo que constituyen un filón todavía poco conocido. Las más interesantes parecen ser los tratados de hisba, los formularios notariales y las fetuas, donde entre otros figuran datos relativos a materiales, técnicas, dimensiones y circunstancias y contextos de las edificaciones.

En cuanto a las fuentes arqueológicas, estudiables a través de la arqueología son desde luego las únicas totalmente fiables a la hora de investigar cualquier aspecto relativo a las construcciones en sí. Sin embargo, los datos que aportan de forma expresa acerca de sus creadores, tales 
como sus nombres, sus oficios, etc., proceden de inscripciones y marcas de cantero, fuentes que en última instancia también son arqueológicas.

\section{ALGUNOS DATOS SOBRE ARQUITECTOS Y CONSTRUCTORES EN AL-ANDALUS OMEYA}

Se abren seguidamente dos apartados: el primero pretende trazar un bosquejo general acerca de los términos empleados para denominar a los trabajadores y la jerarquía de estos en el proceso de ejecución de una obra; en el segundo recogemos algunas noticias concretas, ordenadas cronológicamente, ilustrativas de cuanto se haya dicho.

\subsection{De terminología y jerarquias}

La nomenclatura de los trabajadores de la construcción no está clara en las fuentes, que aportan diversos términos: banna' (de donde "albanil»), sani', pl. sunna' ("artesano»), rakhkham ("marmolista»), naqqash ("escultor», «tallista»), 'arif, pl. 'urafa' (de donde “alarife»)... eran empleados para designar a oficiales y peones, en algunos casos evidentemente especializados, aunque en otros este punto no es comprobable. Por encima se encontraría el muhandis ("técnico", "geómetra", «ingeniero"), que en ocasiones aparece combinado con ellos: al-muhandis al-banna', muhandis al-banna'in, muhandis al-'urafa'... La imprecisión, al menos desde nuestro punto de vista, queda manifiesta desde el momento en que a veces se llega a calificar de varias maneras a un mismo individuo. Las nomenclaturas sólo pueden servirnos, en última instancia, a manera de guía general.

Según Ocaña, los llamados 'urafa' al-banna'in wa s-sunna' son «aquellos albañiles y artesanos que, por los relevantes méritos contraídos en el ejercicio de sus respectivas profesiones, eran distinguidos por sus propios colegas con el preciado galardón de 'arif (cunocedor, maestro, perito)". Los 'urafa' al-muhandisin, "alarifes de los técnicos", solian estar vinculados a la realización de proyectos de obras de gran evergadura.

El nazir al-bunyan era el inspector de la edificación. El número de estos inspectores estaba en razón directa de las dimensiones y la importancia de cada obra.

El sahib al-bunyan era el "jefe de la edificación", director facultativo de toda construcción de carácter oficial. 
El sahib al-abniya (pl. de bunyan) o «jefe de construcciones» tenía jurisdicción sobre todas las obras estatales. Generalmente regentaba tal jefatura por su condición de visir o de hajib (chambelán). Jerárquicamente estaba por encima del nazir y del sahib al-bunyan.

Las construcciones oficiales eran promovidas por el propio soberano, quien se situaba, lógicamente, por encima de los personajes que ostentaban los cargos mencionados. No nos cansaremos de repetir que "todo soberano es constructor" y que las fuentes, en especial las escritas bajo su patronazgo o el de su dinastía, le atribuyen obras de forma más o menos directa. Nos encontramos así ante un tópico vinculado a la ya mencionada relación cerrada poder/construcción. A raíz de ello, la mención de los artistas queda eclipsada tras la «dirección honorífica» del soberano y sus agentes, que es de quienes queda clara constancia, ya que "los arquitectos eran considerados como meros maestros de obras aventajados, que descollaban sobre sus compañeros de profesión, y a quienes pocas veces se dispensaban honores especiales, por lo que sus nombres se perdían, casi siempre, en el anonimato" (Ocaña). Esto es especialmente evidente en el caso de «fundaciones» y reconstrucciones de asentamientos, sean estos grandes o pequeños.

Seguimos recordando y citando a Ocaña: si la obra tenía lugar en Córdoba, capital del Estado, la edificación era dirigida por el sahib al-abniya. Pero "cuando la construcción se realizaba en una provincia, lo corriente era que el sahib al-abniya delegase la dirección nominal de la obra en el 'amil o gobernador de la correspondiente comarca... En las inscripciones fundacionales de la época, no falta nunca la mención del sahib alabniya correspondiente, que es el personaje nombrado a continuación de la consabida frase fatamma bi-awni Allahi 'ala yaday "y se terminó con el auxilio de Allah bajo la dirección de...", como si el mismo fuese, realmente, el auténtico director técnico de la obra en cuestión, lo que ha sido motivo de que se hayan confundido ambas jefaturas modernamente. $Y$, por el contrario, son contados los textos de fundación en que se mencione un sahib al-bunyan de manera precisa y terminante».

La verdad es que no hay que remontarse a fechas tan lejanas para observar este sistema de plasmación de unos nombres en detrimento de otros: las noticias escritas, televisivas o radiofónicas y las placas conmemorativas de obras y monumentos oficiales actuales mencionan al jefe del Estado o del Gobierno, a los gobernadores o alcaldes, a los personajes $u$ organismos promotores... pero raramente a los arquitectos, y menos aún a los aparejadores, delineantes, maestros, oficiales y peones. 
La "estratigrafía jerárquica» descrita podría representarse así:

Alta dirección (nominal u honorífica):

Emir o califa

Gobernador o delegado

Sahib al-abniya

Dirección efectiva o técnica:

Sahib al-bunyan

Inspección de obra:

Nazir al-bunyan

Ejecución de obra:

'Urafa' al-banna'in - Urafa' al-muhandisin - 'Urafa' as-sunna' Banna'un - Sunna' - Naqqashun.....

Las obras de inciativa privada eran promovidas por los propios particulares, quienes contrataban a los trabajadores directamente y según sus posibilidades económicas. La principal base de datos de este tipo de acciones se encuentra de momento en la epigrafía, aunque las fuentes jurídicas parecen prometer al respecto.

\subsection{Noticias concretas}

Presentamos a continuación una serie de noticias procedentes de nuestro "fichero particular». No son todos los datos conocidos ni todos los de nuestro "fichero", sino una selección de entre los que consideramos más representativos. Por lo que se refiere a "fundaciones" o reconstrucciones de asentamientos, sólo las consignamos cuando hay de por medio mención de quiénes las hicieron y en calidad de qué.

Una de las primeras noticias sobre arquitectos en al-Andalus viene dada por el historiador ar-Razi, citado en la crónica titulada Al-Muqtabis, compilación hecha por Ibn Hayyan: allí se menciona a Razin al-Barnasi, un bereber que entró en al-Andalus con el ejército de Tariq en 711. No sólo fue el primer proyectista (mukhatitt) del palacio de recreo de la Ruzafa, 
cerca de Córdoba -iniciativa personal del primer emir de al-Andalus, 'Abdarrahman I (756-88)-, sino que se le atribuyen numerosas obras en la propia capital y en otros lugares, entre ellas la mezquita que lleva su nombre en el arrabal occidental cordobés, unos jardines en el mismo, etcétera.

No sabemos de datos acerca de los constructores de la fase fundacional de la mezquita aljama de Córdoba, monumento señero de la arquitectura omeya andalusí. Es evidente que el tabi'i Hanash y su compañero o compañeros, que entrarian en la Península junto con Musa b. Nusayr, no trabajaron en ella ni en las de Elvira y Zaragoza, como pretende una leyenda bastante extendida. Las fuentes fiables recogen unánimemente que el proyecto inicial de la cordobesa fue concebido por el emir 'Abdarrahman I, quien sin duda debió contar con un arquitecto sirio, ya que el edificio es un claro trasunto de las mezquitas omeyas orientales.

Diversas fuentes (Fath al-Andalus, Nath at-tib y Al-Bayan al-mugrib) se hacen eco de que el emir Hisham I (788-96) se ocupó personalmente de las obras de reparación del puente de Córdoba.

Tudela sería «fundada» en tiempos del emir al-Hakam I (796-822) y de la mano de uno de sus más fieles vasallos, 'Amrus b. Yusuf. El hecho tendría lugar entre los últimos años del siglo vill y los primeros del ix.

La más antigua inscripción andalusí conocida conmemora la construcción de la primera mezquita aljama sevillana: el edificio fue hecho por orden del emir 'Abdarrahman II (822-52) «bajo la dirección de 'Umar b. 'Adabbas, cadí de Sevilla, en el año 214/11 marzo 829-27 febrero 830. Escribió 'Abdalbarr b. Harun". Tenemos ahi una orden emiral, un delegado -el gobernador de la ciudad con el cargo de cadi y que figura como director nominal del trabajo- y un escriba profesional que se encargó de dejar constancia del acto en un epígrafe a modo de «acta fundacional» debidamente fechada. Un documento muy completo.

La inscripción fundacional de la alcazaba de Mérida conservada en el Museo Nacional de Arte Romano y que lleva por fecha rabi" II 220/4 abril2 mayo 835 dice que la obra (bunyan) fue hecha por orden del emir 'Abdarrahman II "bajo la dirección de su 'amil Abdallah b. Kulayb b. Tha'laba y de Jayfar b. Mukassir, su mawla, sahib al-bunyan". Queda documentada una iniciativa emiral y su ejecución con una dirección honorífica -la del gobernador con el cargo de amit y una efectiva, la del "jefe de construcciones".

En el año $230 / 18$ septiembre $844-6$ septiembre 845 se hicieron obras en la muraila de Sevilla. De ellas se ocupó el sirio 'Abdallah b. Sinan, cuyo 
nombre fue grabado en las puertas de la cerca. Transmite los datos lbn alQutiyya.

Entre las noticias de la ampliación de la mezquita aljama de Córdoba por 'Abdarrahman II, ampliación concluida por su hijo Muhammad I (852. 86), aparecen tres ejemplos de nazir al-bunyan o inspector de obras: los fatas Nasr y Masrur y el zabazala Muhammad b. Ziyad. En el dintel de la puerta de San Esteban en esa mezquita figura escrito: "Ello [la obra] se terminó en el año 241/22 mayo 855-9 mayo 856, con la bendición de Dios y Su ayuda, [bajo la dirección de] Masrur, su fata". Este último "su" se refiere, claro está, a Muhammad I. La inscripción está documentada, aunque no transcrita, por Ibn 'Idhari. Ibn Hayyan atribuye implícitamente el cargo de inspector al emir Muhammad, ya que tras la conclusión de las obras entró en la mezquita a solas con sus principales hombres, la recorrió recreándose en lo que alii se había hecho, hizo humildemente sus oraciones y se volvió al alcázar. En cierto momento de su emirato, no sabemos precisamente cuándo, hubo amenaza de ruina en el edificio. Muhammad 1 envió al hajib, a los visires y a los jefes de construcciones (ashab al-bunyan) "para que viesen con sus propios ojos" lo que allí ocurría: sus dos muros (sic) se habian inclinado, sus jácenas se habian roto y la cubierta estuvo a punto de ceder, siempre según la fuente. Los jefes de construcciones se las ingeniaron para que aquello no se desplomara e hicieron unos soportes a fin de sujetar los dos muros, que con eso aguantaron laslluvias de aquel invierno.

Ibn Hayyan es e! primero que recoge la [re]construcción de Esteras, Madrid, Peñafora y Talamanca por iniciativa personal de Muhammad I. Fuentes posteriores se hacen eco de ello, pero ninguna aporta fechas precisas.

En 239/12 junio 853-1 junio 854 al-Hakam, hermano del emir Muhammad, salió en plan de aceifa, hizo alto con el ejército en Calatrava, cuidó de [re]hacer su muralla $y$ fortificar su entorno, trajo de vuelta a sus habitantes - que la habian abandonado-y la dotó de una guarnición. Lo mismo hizo con la muralla de Talavera y el hisn de Jándula. Tenemos noticia de estos hechos a través de Ibn Hayyan, Ibn 'Idhari y la Descripción Anónima.

Relata el Bayan de Ibn 'Idhari que en 242/10 mayo 856-29 abril 857 Musa b. Musa b. Qasi, señor (sahib) de Zaragoza, amplió la mezquita aljama de su ciudad con la quinta parte del botín logrado al conquistar el hisn cristiano de Tarrasa.

Las noticias sobre constructores también involucran a estos en obras destructivas: nadie mejor que un constructor para saber cómo demoler. 
En el año 244/19 abril 858-7 abril 859, en el contexto de una campaña contra Toledo, el emir Muhammad ordenó destruir su puente -el actualmente llamado "de Alcántara" - como parte de una estratagema: "congregó así a los alarifes de los albañiles y los técnicos (al-'urafa' mina l-banna'in wa l-muhandisin), quienes dirigieron el plan desde donde no se apercibian los toledanos. A continuación se retiraron de allí, y cuando los rebeldes se juntaron en el puente, este se quebró con ellos y sus partes se desplomaron, cediendo con los valedores y campeones que sobre él estaban, quienes hasta el último se ahogaron en el río. Aquella fue una de las cosas más terribles que Dios les hizo", según Ibn 'Idhari.

Ibn Hayyan y al-'Udhri recogen datos sobre la creación de la madina de Calatayud: en 248/7 marzo 862-23 febrero 863 el emir Muhammad instaló allí a 'Abdarrahman b. 'Abdal'aziz at-Tujibi, cabeza de un clan militarizado y unido a los Omeyas por vínculos de fidelidad. 'Abdarrahman [re]construyó Calatayud, Daroca, Somed y Furtish, realizando con ello una acción parecida a la de 'Amrus b. Yusuf en Tudela más de medio siglo antes. En ambos casos habría que pensar que las obras, de inciativa emiral y encargadas a un jefe militar, serian hechas por las propias tropas dirigidas técnicamente por especialistas. Esta consideración es extensible a todas 0 prácticamente todas las construcciones castrenses.

Según Ahmad b. Muhammad ar-Razi, a través del Muqtabis de Ibn Hayyan, el emir Muhammad creó su propia finca de recreo, la almunia de Kintush. El Muqtabis considera largamente el hecho y las consecuencias de ser esa almunia un proyecto personal del emir. No fue su única construcción civil, ya que en 250/13 febreo $864-1$ febrero 865 hizo numerosas obras en el gran alcázar y en las almunias exteriores al mismo, según lbn 'Idhari.

En dhu I-qa'da del año 250/4 diciembre 864-2 enero 865 fue [re]construida la mezquita aljama de Madinat Ilbira, cerca de Granada. "Ordenó [re]construirla el emir Muhammad... y se terminó con el auxilio de Dios bajo la dirección de 'Abdallah b. 'Abdallah, su 'amil de la cora de Elvira", según reza una inscripción perdida y que se transmite a través de la Ihata, crónica cuyo autor es Ibn al-Khatib.

En el año 261/16 octubre 874-5 octubre 875 Ibn Marwan al-Jilliqi pidió el amán del emir Muhammad y su permiso para instalarse en Badajoz, entonces vacía. Ibn Marwan [re]construyó Badajoz y la consolidó como ciudad. Estos hechos están recogidos en distintos términos por varias fuentes. Al-Bakri, geógrafo del siglo XI, dice por su parte que Ibn Marwan solicitó del emir 'Abdallah (888-912) el envio de obreros (fa'ala) a fin de que le contruyeran una mezquita aljama y un baño. Según Torres Balbás, 
esta noticia permite ver que Córdoba era "centro en el que estaban concentrados los obreros y artistas de la construcción, como indica su envío por el emir...». Sin embargo, ello no obsta para que hubiera escuelas y grupos locales por todo al-Andalus: nos encontramos ante unas acciones de carácter oficial u oficialista, de ahí el envio directo, por lo que no deben plantearse conclusiones tajantes al respecto. Veremos algún otro caso.

También en 261/874-5 tuvo lugar la [re]construcción de la muralla de Huesca por el valí de esa ciudad, 'Amrus b. 'Umar b. 'Amrus b. Yusuf, nieto del 'Amrus de Tudela, dato significativo. Al-'Udhri transmite la noticia junto con parte de la inscripción colocada sobre una de las puertas. Decía dicha inscripción: “... de lo que [re]construyó Khafif el constructor (a/banna) bajo la dirección de 'Amrus b. 'Umar, 'amil del imán Muhammad b. 'Abdarrahman, Dios le guarde por su fidelidad".

En el año 266/23 agosto 879-11 agosto 880 el príncipe 'Abdallah, hijo del emir Muhammad, salió en campaña contra las coras de Rayya y Algeciras. Allí [re]construyó fortalezas donde situó a las gentes obedientes para que se hiciesen cargo de sus algaras. Entre esas fortalezas se contaba la de Cardela (?). El hecho está recogido por Ibn Hayyan e Ibn 'Idhari.

Hashim b. 'Abdal'aziz, notable individuo, fue ascendido a visir y nombrado valí de la cora de Jaén durante el mandato del emir Muhammad. Ibn Hayyan precisa que bajo la dirección de este Hashim se [re]construyeron Úbeda y la mayor parte de "las fortalezas inexpugnables» de esa cora.

La iniciativa constructiva no sólo estuvo en manos masculinas, sino que también hubo mujeres mecenas de la arquitectura. Los datos disponibles corresponden a grandes personajes de la corte cordobesa o a destacadas figuras de las provincias. Un caso preclaro a este respecto fue Marjan, esposa de 'Abdarrahman III an-Nasir (912-61, califa desde 929), de quien a través de Ibn Hayyan se sabe construyó mezquitas, sin que se tengan noticias más precisas.

Era muy frecuente, al poner asedio a una ciudad o a una gran fortaleza, que esta se resistiera durante meses e incluso años. En tales casos se hacía necesario establecer un campamento (mahalla) o una fortificación donde mantenerse los sitiadores. 'Abdarrahman 111 mandó construir la no localizada sakhra de 'Udan en la primavera del año 919 para reducir al rebelde Ja'far b. 'Umar b. Hafsun, que estaba en Balda (Antequera?).

Entre julio de 922 y mayo de 927 'Abdarrahman III ordenó erigir forticaciones de asedio contra Bobastro. Ibn Hayyan documenta, entre otras, Sakhrat al-Madina, no localizada, y el campamento (mahalla), también lla- 
mado hisn y madina, de Taljayra, hecho a su vez sobre un hisn anterior. Se ha identificado con la fortificación de “El Castiliejo", próxima a Bobastro. Una vez tomada la ciudad, ordenó hacer construcciones diversas en su alcazaba. Comenzaron en enero de 928 y la inspección personal del soberano tuvo lugar en noviembre de 929, cuando aún no habían finalizado las obras.

Del mes de muharram del año $318 / 3$ febrero-2 marzo 930 data la inscripción conmemorativa de una siqaya (muy posiblemente una fuente) en Écija. Su iniciativa corrió a cargo de Abdarrahman III, califa desde hacía poco más de un año. Como director honorífico consta su mawla y 'amil Umayya b. Muhammad b. Shuhayd. Aparece además el nombre del constructor, “Fath, gulam (siervo) del Emir de los Creyentes», quien como rakhkham ("marmolista") figura en un capitel sin fecha, a nombre de 'Abdarrahman III, correspondiente a una obra hecha bajo la dirección de Shunayf, fata de este soberano.

Acabamos de mencionar un capitel epigrafiado. $Y$ es que son frecuentes las inscripiones sobre piezas arquitectónicas, gracias a las cuales poseemos información de gran interés. La primera que citaremos aquí con fecha expresa (320/13 enero-31 diciembre 932) se encuentra en uno de los capiteles conservados en el Salón de Embajadores del Alcázar de Sevilla. Se menciona al califa 'Abdarrahman III y al autor. Ahora bien, ¿autor de qué?: ¿Del capitel?, ¿de la obra donde este estaba?... Es difícil saber exactamente qué papel desempeñó el personaje, pues lo que dice la inscripción es "De lo que fue hecho bajo la dirección de Shunayf, su fata", mencionado en el párrafo anterior.

Mientras se hacian fuentes y elementos arquitectónicos de gran belleza se construía Madinat al-Fath, "La Ciudad de la Victoria", en el monte Chalencas. La mandó erigir el califa en persona para poner sitio a los toledanos entre junio y julio de 930. Volvió a ser utilizada por él en agosto de 932, ocasión en que se le llama «campamento urbanizado» y «triunfante campamento».

En el verano de ese mismo año el califa reparó el puente «de Alcántara» y ordenó construir el Alcázar y el "Ceñidor» de Toledo.

En el caso del asedio de Zaragoza, prolongado de 935 a 937, an-Nasir hubo de ordenar la erección de un campamento en el lugar conocido como al-Jazira. Ibn Hayyan, al-'Udhri e Ibn Khaldun hablan de las construcciones que el soberano mandó hacer alli, todas ellas según su parecer personal.

El 12 de julio de 936 hubo un incendio en el zoco de Córdoba, que fue reconstruido a instancias de Abdarranman III. Con el zoco en sí Ibn 
Hayyan menciona otras dos obras concretas: la mezquita de Abu Harun casi sin duda el onomástico de su fundador-y el edificio de las postas. Es de señalar que zoco, mezquita y edificio de postas son tres lugares públicos de importancia primordial en la estructura de una ciudad islámica, máxime tratándose de la capital del Estado. De ahí que el califa tome personalmente las riendas de su reconstrucción.

El volumen $V$ del Muqtabis contiene un interesantísimo pasaje acerca de trabajadores de la construcción. Aunque se refiere a obras realizadas en Marruecos, sus agentes eran andalusíes y es evidente que trabajaban para el califa, quien los envió a su aliado magrebí Musa b. Abi l-Afiya para "[re]construir el castillo de Jara" en el año 936. A tal fin "an-Nasir le mandó de su parte a Muhammad $b$. Walid b. Fushtayq, su protoarquitecto (ra'is al-muhandisin), con 30 albañiles (banna), 10 carpinteros (an-najjarin), 15 cavadores (al-haffarin), seis hábiles caleros (al-jayyarin al-muhsinin li-amali l-jir), seis aserradores de madera (al-ashsharin li-ashri l-khashab), dos herreros (rajulayn mina I-haddadin) y dos estereros (rajulayn mina Ihassarin), escogidos entre los más hábiles de su profesión, acompañados de cierto número de herramientas y accesorios para los trabajos que ejercían, todo lo cual le hizo llegar el sultán para superar el período que durara el trabajo requerido...". Hacia el verano de 937 Musa b. Abi l-'Afiya volvió a escribir al califa de Córdoba: «Pedía la sustitución de los albañiles y operarios (al-banna'in wa l-fa'ala) andalusies por otros más activos, pues estaban aburridos del trabajo y se les hacía larga la ausencia de su país".

Entre junio y julio de 937 'Abdarrahman lil ordenó la consolidación de cuantas fortalezas, torres y atalayas eran débiles o estaban dañadas entre Atienza y Talavera. No se especifica el nombre de ninguna de ellas. El hecho se inscribe en un plan de refuerzo general de la Frontera Media dirigido por el propio califa, quien a finales de ese mismo año hizo algo similar entre Lérida y Atienza, territorio que comprende gran parte de la Frontera Superior y su enlace con la Media. Se trata de dos acciones complementarias, lo que el propio texto de Ibn Hayyan señala. Tampoco en esta última ocasión se identifica ninguna fortaleza concreta.

Bajo la dirección honorífica de un mawla de 'Abdarrahman III, el visir y zalmedina Abdallah b. Badr, fueron concluidas las obras de un qanat en Córdoba el último día de safar del año 329/3 diciembre 940.

Madinat az-Zahra', ciudad áulica furıdada por 'Abdarrahman III ya califa, constituye la versión secular del gran proyecto arquitectónico de representación del poder califal cuya faceta religiosa es la ampliación de la mezquita aljama de Córdoba -ejecutada por su hijo al-Hakam II-. Característica, pues, de esta ciudad-palacio son sus iniciativa, proyecto y 
dirección horífica personales del soberano. Las fuentes se hacen eco, más - menos literariamente, de este asunto: dirigieron las obras el propio 'Abdarrahman III y su hijo el entonces príncipe al-Hakam. En su diccionario biográfico, Ibn al-Faradi apunta que en Madinat az-Zahra' trabajaron mil obreros: trescientos albañiles, doscientos carpinteros y quinientos peones. Mil obreros mencionan también la Descripción Anónima y al-Maqqari. Este último e Ibn Galib aportan el nombre del arquitecto supervisor de sus obras (al-muhandis an-nazir fi bunyani-ha): Maslama b. 'Abdallah. Los cronistas Ibn Khaldun y al-Maqqari dicen que el califa "hizo venir a los alarifes de los técnicos y los albañiles ('urafa' al-muhandisin wa l-banna'in) de todas partes, y le llegaron hasta de Bagdad y Constantinopla". Al Maqqari dice asimismo que el mármol para la construcción de esta ciudad fue traído de Cartago, Ifriqiya y Túnez por 'Abdallah b. Yunus, alarife de los albañiles ('arif al-banna'in) y Hasan y 'Ali b. Ja'far al-Iskandarani ("el Alejandrino»). Se les pagaba por cada pieza.

Los datos epigráficos arrojan mucha luz sobre los constructores de Madinat az-Zahra': de momento sabemos de unas 60 inscripciones constructivas publicadas procedentes de varios puntos de esta ciudad-palacio, fechadas entre 333 y 364/24 agosto $944-9$ septiembre 975. Por ceñirnos sólo al "Salón Rico" o "Salón de 'Abdarrahman III», objeto de un reciente estudio epigráfico de $M^{a}$ Antonia Martínez y de reflexiones de $A$. Fernández-Puertas, citaremos en primer lugar a sus ashab al-abniya, que son tres: Shunayf, 'Abdallah b. Badr y Ja'far. Por debajo de ellos aparecen Aflah, Badr, Fath, Galib b. Sa'd, Muhammad b. Sa'd, Muzaffar, Nasr, Rashiq, Sa'd, Sa' id al-Ahmar, Sa' id b. Fath y Tarif. Tras cada uno de estos últimos, la palabra 'abdu-hu (su siervo) y a veces el oficio: ar-rakhkham («el marmolista») o an-naqqash («el escultor»). Se trataría de "siervos escultores" procedentes de los talleres oficiales y quizás a la vez servidores de la corte con cargos más o menos hereditarios, como indican sus filiaciones. Varios trabajaron en el alcázar y en la mezquita aljama de Córdoba, pero los nombres de algunos de ellos aparecen también en "piezas oficiales» de marfil, metal y cerámica (!), lo cual ha llevado a plantear cuál fue el papel efectivo de esos «siervos artistas» en Madinat az-Zahra', pues su "ubicuidad" hace pensar que debia tratarse, más que de ejecutores de los trabajos en sí, de una especie de "encargados de obra" o, más aún, de auténticos "directores (ashab) de la dar as-sina'a", siempre por debajo de Shunayf, 'Abdallah b. Badr y Ja'far. La cuestión es interesantísima, sin duda.

Obras de iniciativa particular de an-Nasir fueron las correspondientes a los alcázares edificados para residir sus hijos. No se citan cronologías precisas, pero el último debió quedar concluido entre junio y julio de 943. 
En el año 333/24 agosto 944-12 agosto 945 tuvieron lugar las obras correspondientes a las atarazanas o arsenal (ad-dar as-sina'a) de Tortosa. En la inscripción conmemorativa figuran la iniciativa del califa, la dirección honorífica de su cadí y siervo ('abd) 'Abdarrahman b. Muhammad y el nombre de quien escribió (kataba): 'Abdallah b. Kulayb.

Del primer mes del mismo año data una de las inscripciones procedentes de la rábita de Guardamar del Segura (Alicante): dice que "se acabó esta mezquita en el mes de muharram del año 333/24 agosto-22 septiembre 944 . Ordenó su [re]construcción Ahmad b. Bahlul b. Thabit... bajo la dirección de Muhammad b. Abi Salama [y] 'Umar b. Makhlad Abi I-'A...”. Una iniciativa privada, pues, y un director quizás no tan honorífico como real.

Hay varios capiteles que mencionan a un director de obra, 'Atiq, fata y mawla de 'Abdarrahman III. Están fechados entre 340 y 342/9 junio 951-6 mayo 954. En uno de ellos figura quien quizás fuese el tallista de la pieza, cuyo nombre emprezaba por $>r<y$ terminaba por $>y<$; en otro, Saliq el marmolista (rakhkham).

De Arcos de la Frontera (Cádiz) o de sus alrededores procede una inscripción conmemorativa de la erección de una mezquita y un alminar por iniciativa privada: los construyó (bana-huma) 'Attab b. Harun b. Nasr. Está fechada en ramadán del año 340/31 enero-29 febrero 952.

Director honorífico de las obras de consolidación de la fachada que da al patio de la mezquita aljama de Córdoba en el mes de dhu I-hijja del año 346/23 febrero-24 marzo 958 fue el visir y zalmedina de la ciudad 'Abdallah b. Badr, como atestigua la inscripción del Arco de las Palmas en dicho edificio. Firma la lápida Sa'id b. Ayyub.

En el castillo de Tarifa hizo obras 'Abdarrahman III. Según la inscripción allí conservada, ordenó la [re]construcción de un burj, lo que fue terminado en safar del año 349/2-30 abril 960 «bajo la dirección de su visir 'Abdarrahman b. Badr, su mawla”.

349/3 marzo 960-19 febrero 961, sin más precisiones, es el año en que fue labrado un arquito de una sola pieza que se conserva en el claustro de la Catedral de Tarragona. En él aparece el promotor de la obra -el califa- y el director de la misma, Ja'far, su fata y mawla, a quien ya hemos visto y no tardaremos en volver a ver.

Ya del período de al-Hakam II (961-76) es un capitel conservado en la casa de los Sres. de Herruzo Sotomayor (calle Cabezas, 5), en Córdoba. Conmemora "lo que mandó hacer [el califa] y se acabó, con la ayuda de Dios, bajo la dirección de su mawla, hajib y sayf ad-dawla Ja'far b. 'Abdarrahman, para los aposentos del Alcázar en el año 353/19 enero 964-6 
enero 965». Casi idénticas inscripciones llevan otros conservados en el Museo Arqueológico de dicha ciudad. Uno de ellos especifica, en la cara superior del ábaco, que es "Obra de Safar para la mezquita de su señor». Vemos que Ja'far ya no sólo es mawla y hajib, sino que ahora posee también el título honorífico de sayf ad-dawla, "espada del Estado". En otros dos capiteles de la misma serie aparece como mawla, hajib y katib, "secretario".

De la ampliación de la mezquita de Córdoba por al-Hakam II hay abundantes noticias de nuestro interés: el califa aparece como "jefe supremo" del proyecto hasta el punto de haber pretendido desviar la alquibla para orientarla correctamente hacia La Meca, idea de la que no desistió sino tras habérsela desaconsejado numerosos sabios. El director honorífico de las obras fue Ja'far b. 'Abdarrahman. Encargados de la inspección (nazr) fueron los ashab ash-shurta (jefes de policía) Muhammad b. Tamlikh, Ahmad b. Nasr y Khald b. Hashim, así como el katib (secretario) Mutarrif b. 'Abdarrahman. Ja'far b. 'Abdarrahman, mawla del califa, contaba con los títulos de hajib y sayf ad-Dawla, según datos de las inscripciones y de la crónica Al-Bayan al-Mugrib. Importantes personajes visitaron las obras: así el cadí Mundhir b. Sa'id, acompañado del encargado de obras pías, de juristas y de notarios (al-'udun).

En las escalas «inferiores» de la construcción de esa ampliación también encontramos nombres propios en forma de firmas: en la cornisa de mármol del nicho del mihrab figuran como autores Fath, Tarif, Nasr y Badr. También hay marcas de identidad en basas, fustes, capiteles y cimacios: entre ellas están las firmas de Aflah, Badr, Nasr, Ibn Nasr, Qasim y Rashiq. Vemos que la gliptografía entra en acción como ciencia auxiliar para hacer la historia de la construcción y los constructores en este ámbito. $Y$ que gracias a ella quedan documentados algunos de los artistas que ya habian trabajado en otras obras de iniciativa oficial.

Maysur b. al-Hakam, mawla y caíd del califa al-Hakam II —de quien tomó su filiación-, está documentado epigráficamente como director de las obras de [re]construcción de un burj situado con muchas probabilidades en el solar que ocupa la actual fortaleza de Baños de la Encina (Jaén), edificio de época almohade. La inscripción, conservada en el Museo Arqueológcio Nacional, lleva como fecha el mes de ramadán del año $357 / 31$ julio-29 agosto 968 .

Del año 358/25 noviembre 968-13 noviembre 969 data la inscripción correspondiente a un edificio religioso cordobés hecho por orden de alHakam II. Se concluyó «con el auxilio de Dios y Su poder, bajo la dirección de su mawla y hajib Ja'far b. 'Abdarrahman - iDios esté satisfecho de él!-, con la inspección de Ma'qil y Tammam, sus fatas". 
Varios capiteles de los conservados en el Alcázar sevillano documentan la construcción de una mezquita en el año 363/2 octubre 973-20 septiembre 974. En una cartela del ábaco de uno de ellos reza "Obra de Fath". ¿Se trata de aquel gulam de 'Abdarrahman III que figuraba en la siqaya de Écija de 318,45 años antes, y a quien hemos visto pasar por Madinat az-Zahra' y la mezquita aljama de Córdoba, donde volverá a aparecer? De ser así, habría que convenir que "hizo carrera" a lo largo de su vida.

Mecenas de un alminar, una galería aneja a él y las decoraciones de la mezquita correspondiente -que estaría en el solar de la actual iglesia de San Lorenzo de Córdoba - fue Mushtaq, madre de al-Mugira, hermano paterno de al-Hakam II. La obra fue dirigida por el conocido 'Atiq b. 'Abdarrahman, su fata, y se terminó en un mes de ramadán entre los años 360 y $365 / 28$ junio $971-1$ junio 976 . Los datos figuran en una inscripción conservada en el Museo Arqueológico de Córdoba.

La inscripción de una obra realizada bajo el califato de Hisham II ostenta el nombre de este a la vez que deja claro quién fue la mecenas: se trata de la conmemoración de la [re]construcción de una fuente (?) (siqaya) en Écija mandada hacer por la madre del califa y «bajo la dirección de su [de ella] sani'a, sahib ash-shurta (jefe de polícía) y cadí de las gentes de la cora de Écija y Carmona y sus 'amales, Ahmad b. 'Abdallah b. 'Arus. Ello tuvo lugar en el mes de rabi' II del año $367 / 16$ noviembre-14 diciembre 977". ¿Remodelación del monumento que había hecho su suegro 'Abdarrahman III en 318?

Muhammad b. Abi Amir, Almanzor, usurpador del poder de los Omeyas, tomó también las formas plásticas de expresión del mismo, por lo que fue un gran constructor pendiente siempre de la marcha de sus obras y del estado de las edificaciones: "Al regresar de sus campañas no descansaba hasta haber convocado [y consultado]... al jefe de construcciones (sahib al-abniya) cuando amenazaban ruina alguno de sus muros, sus edificios, sus alcázares o sus casas", según testimonio de al-Maqqari.

Por supuesto, Almanzor escenificó su poder y emuló a sus legítimos antecesores ampliando la mezquita aljama de Córdoba - las obras debieron empezar en 377/mayo 987-20 abril 988- y trabajando personalmente en ella, según el citado al-Maqqari. Su director honorífico fue 'Abdallah $b$. Sa'id b. Muhammad b. Batri, sahib ash-shurta de Córdoba. En basas, fustes, capiteles y cimacios se encuentran varias marcas de identidad, como ocurría en la zona de al-Hakam II: las epigráficas árabes adoptan las grafías correspondientes a los nombres Aflah, Aflah al-Qurra, 'Amir, Bushra (?), Durri, Faraj, Fath (iel mismo otra vez?), Hakam, Khalaf, Khalaf al'Amiri, Khayra, Kah, Mas'ud, Maysur, Mubarak, Mubarak b. Hisham, Nasr, 
Rizq, Sa'ada y Yahya. Khalaf al-'Amiri debía ser esclavo o liberto personal (mawla) de Almanzor, de ahí su filiación; lo mismo cabe decir de Mubarak b. Hisham respecto del entonces califa. Los nombres de Mas'ud, Mubarak y Nasr - ya presentes en la ampliación de al-Hakam $11-$, cuyas traducciones latinas son respectivamente “Felix», "Benedictus» y "Victor», han hecho pensar que se trataba de artesanos cristianos procedentes de la mozarabía de Córdoba, a quienes no hay que confundir con los cristianos cautivos que se sabe trabajaron en las obras de Almanzor y cuyas labores no serían las especializadas de talla y escultura de elementos, sino más bien otras de carácter ingrato. Reforzaría esta hipótesis de artistas cristianos la presencia de marcas consistentes en un áncora, una tau -forma estilizada de cruz-, una barca, un grano (¿de mostaza?), un gancho o anzuelo, una pentalfa y una hexalfa (¿estrella matutina?), asi como las letras latinas $>1<,>\mathrm{O}<,>\mathrm{S}<\mathrm{y}>\mathrm{E}<$. Además de todas estas marcas, también aparecen signos más o menos abstractos: $\S$ ("enlace» de $>\mathrm{O}<\mathrm{y}>\mathrm{S}<$ ), (doble $>\mathrm{O}<$ ) y $\|$, aislados o combinados entre sí. Algunos de estos signos también figuran en la ampliación de al-Hakam II.

Almanzor no se contentó con ampliar la mezquita aljama de la capital del Estado, sino que también quiso tener, como los emires y califas a quienes imitaba, su propia ciudad palatina, a la que llamo Al-Madina az-Zahira, en realidad un homófono de Madinat az-Zahra'. Aparte de ser «su» creación, con todo lo que esto implica en el sentido de ser él el "arquitecto supremo", Ibn "Idhari, al-Himyari y al-Maqqari documentan que para hacerla "congregó a artesanos y peones y trajo máquinas magníficas (hashada s-sunna' wa l-fa'ala wa jalaba ilay-ha l-alata l-jalila)».

El gran usurpador ordenó realizar obras en el puente «de Alcántara» de Toledo. "La noticia nos ha llegado indirectamente, pero con caracteres de indudable autenticidad, a través de una traducción y repetidas copias del epigrafe original» (Torres Balbás). Dichas obras fueron "hechas» por Khalaf b. Muhammad al-'Amiri, "alcayde" de la ciudad, y están fechadas en 387/14 enero 997-2 enero 998, según los datos de que disponemos. ¿Se trataría del citado Khalaf al-'Amiri, quien firmó varias piezas de la ampliación almanzoreña de la aljama de Córdoba?

El último testimonio que conocemos de una construcción bajo el poder efectivo de los Omeyas andalusies es la inscripción conmemorativa de la mezquita de Al-Bab al-Mardum, hoy iglesia llamada "Cristo de la Luz": “En el nombre de Dios, el Clemente, el Misericordioso. Erigió esta mezquita Ahmad b. Hadidi, de su peculio, solicitando la recompensa ultraterrena de Dios. Se concluyó, con el auxilio de Dios, bajo la dirección de Musa b. 'Ali, el constructor (banna), y de Sa'ada. Fue terminada en muna- 
rram del año 390/13 diciembre 999-11 enero 1000". Como vemos, esta última construcción documentada es de inciativa privada.

\section{FUENTES ÁRABES CITADAS}

- Anónimo (s. XIV), Dhikr bilad al-Andalus (Una descripción anónima de al-Andalus), ed., trad. esp., notas e índices L. Molina, Madrid, 1983.

- Anónimo (s. XII), Fath al-Andalus, ed. L. Molina, Madrid, 1994; trad. esp. y notas J. de González, Argel, 1889.

- Al-Bakri (s. xI), Kitab al-masalik wa l-mamalik, ed. 'A. al-Hajji, Beirut, 1968; trad. esp. E. Vidal Beltrán, Zaragoza, 1982.

- Al-Himyari (ca. s. Xv), Kitab ar-rawd al-mitar fi khabar al-aqtar, ed. $y$ trad. franc. É. Lévi-Provençal, Leiden, 1938.

- Ibn al-Faradi (962-1013), Kitab tarikh 'ulama' al-Andalus, ed. F. Codera y Zaidín, Madrid, 1891.

- Ibn Galib (s. XII), Kitab farhat al-Anfus, ed. L. 'Abdalbadi' en Majallat Ma'had al-Makhtutat, I, 2, 1955, pp. 272-310; trad. parc. esp. J. Vallvé, "Una descripción de España de Ibn Galib", Anuario de Filología, s. n., 1975, pp. 369-84.

- Ibn Khaldun (1333-82), Kitab al-ibar, ed. anónima, Bulaq, 1284/1867-8; trad. esp. O. Machado en Cuadernos de Historia de España, IV, 1946, a XLVII-XLVIII, 1968.

- Ibn al-Khatib (1313-74), Al-Ihata fi akhbar Garnata, ed. M. 'A. 'Inan, El Cairo, 1973-8.

- Ibn Hayyan (988-1076), Kitab al-Muqtabis fi tarikh rijal al-Andalus, vol. II, ed. M. 'A. Makki, Beirut, 1973; vol. III, ed. M. F. Antuña, París, 1937; vol. V, ed. P. Chalmeta, F. Corriente, M. Subh et al., Madrid, 1979, trad. esp. M J. Viguera y F. Corriente, Zaragoza, 1981.

- Ibn 'Idhari (m. 1312), Al-Bayan al-mugrib, vol II, ed. G. S. Colin y É. Lévi-Provençal, reimp. Beirut, 1983.

- Ibn al-Qutiyya (m. 977), Tarikh iftitah al-Andalus, ed. I. al-Abyari, Beirut, 1982; trad. esp. J. Ribera, Madrid, 1926.

- Al-Maqqari (ss. XVI-XVII), Nafh at-tib, ed. I. 'Abbas, reimp. Beirut, 1988.

- Al-'Udhri (1002-86), Kitab tarsi' al-akhbar..., ed. parc. 'A. AlAhwani, Madrid, 1965; trad. parc. esp. Granja, F. de La, La Marca Superior en la obra de al-'Udri, Zaragoza, 1966. 


\section{VOCABULARIO}

- 'abd, pl. 'abid: siervo, esclavo.

- aceifa: campaña militar (de verano).

- algara: campaña militar.

- aljama (mezquita): mezquita congregacional, la principal de todo asentamiento islámico.

- almunia: huerta; finca (de recreo).

- almotacén: inspector de mercados.

- alquibla: muro que, en una mezquita, indica la orientación hacia La Meca.

- amal: obra, trabajo. También «circunscripción territorial».

- 'amil: gobernador de un 'amal (con el sentido de "circunscripción territorial»).

- amán: perdón; tratado de capitulación.

- 'arif, pl. 'urafa': conocedor, maestro, perito. De esta voz deriva el arabismo «alarife».

- ashshar, pl. nominativo -un, pl. genitivo/acusativo -in: aserrador.

- banna', pl. nominativo -un, pl. genitivo/acusativo -in: constructor; albañil.

- bunyan, pl. abniya: construcción, obra constructiva.

- burj: «torre»; por extensión, «fortificación».

- cadí: juez. Quien tenía este cargo actuaba a menudo como gobernador.

- caíd: jefe militar. Gobernador.

- cora: circunscricpión territorial.

- dar as-sina'a: talleres oficiales. También arsenal, atarazanas, dársena.

- dhu I-hijja: mes del calendario islámico.

- dhu l-qa'da: mes del calendario islámico.

- fa'il, pl. fa'ala: operario, obrero, peón.

- fata: esclavo. Eunuco.

- gulam: siervo. 
- haddad, pl. nominativo -un, pl. genitivo/acusativo -in: herrero.

- haffar, pl. nominativo -un, pl. genitivo/acusativo -in: cavador.

- hajib: chambelán; tb. cierto título.

- hassar, pl. nominativo -un, pl. genitivo/acusativo -in: esterero.

- hisba: oficio del almotacén.

- hisn: fortaleza.

- jayyar, pl. numinativo -un, pl. genitivo/acusativo -in: calero.

- katib: secretario.

- madina: ciudad.

- mahalla: campamento militar.

- mawla: liberto; “cliente».

- mihrab: nicho empotrado en la alquibla y que señala la dirección de La Meca.

- muhandis: técnico; geómetra; ingeniero.

- muharram: mes del calendario islámico.

- mukhatitt: lit., "proyectista», persona que realiza un proyecto arquitectónico.

- najjar, pl. nominativo -un, pl. genitivo/acusativo -in: carpintero.

- naqqash, pl. nominativo -un, pl. genitivo/acustivo -in: tallista.

- nazir al-bunyan: lit., «inspector de la construcción».

- qanat: cierto tipo de obra hidráulica.

- rabi“ I/rabi' II: meses del calendario islámico.

- rakhkham: marmolista.

- ra'is: cabecilla; jefe.

- ramadán: mes del calendario islámico.

- safar: mes del calendario islámico.

- sahib, pl. ashab: lit., "señor»; jefe.

- sahib al-abniya : jefe de construcciones con jurisdicción sobre todas las obras estatales.

- sahib al-bunyan: director facultativo de toda construcción de carácter oficial.

- sahib as-shurta: jefe de policía. 
- sakhra: lit., "peña». Cierto tipo de fortificación.

- sani", pl. sunna': constructor (lit., "artesano»).

- sani'a: cliente; protegido.

- sayf ad-dawla: lit., "espada del Estado» (0 "de la dinastía»). Cierto título honorífico.

- siqaya: cierto tipo de obra hidráulica.

- tabi'i: persona perteneciente a la generación siguiente a la del Profeta Muhammad y sus Compañeros.

- 'udul ( $\mathrm{pl}$. de 'adl): testigos instrumentales. Suele traducirse por «notarios».

- valí: gobernador.

- visir: ministro.

- zabazala: director de la oración en la mezquita.

- zalmedina: cierto cargo con autoridad oficial.

- zuna: conjunto de tradiciones relativas al Profeta Muhammad.

EMIRES Y CALIFAS OMEYAS DE AL-ANDALUS

1 Abdarrahman 1756

2 Hisham | 788 H1

3 Al-Hakam I 796 H2

4 'Abdarrahman II 822 H3

5 Muhammad $1852 \mathrm{H} 4$

$6 \mathrm{Al}-$ Mundhir $886 \mathrm{H} 5$

7 Abdallah 888 H5

8 'Abdarrahman III 912 N7 (califa desde 929) 


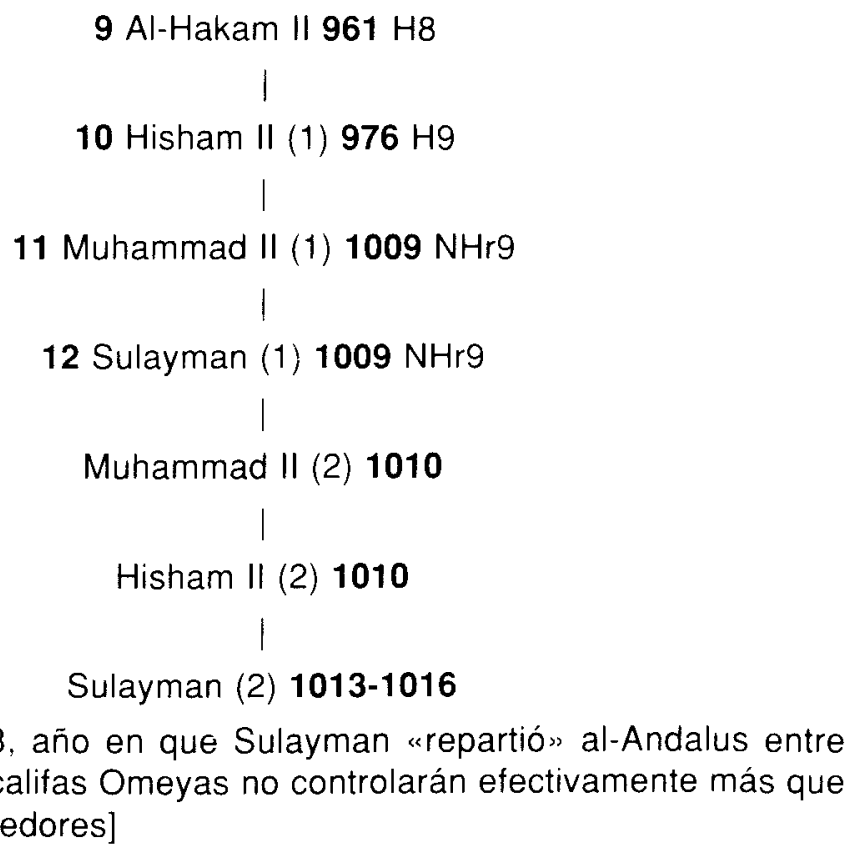

[A partir de 1013, año en que Sulayman "repartió" al-Andalus entre sus partidarios, los califas Omeyas no controlarán efectivamente más que Córdoba y sus alrededores]

\author{
13 'Abdarrahman IV $1018 \mathrm{NHr} 9$ \\ 14 'Abdarrahman V $1023 \mathrm{Hr} 11$ \\ 15 Muhammad III 1024-1025 NHr9 \\ 16 Hisham III 1027-1031 Hr13
}

Claves

$\mathrm{H}$-Hijo
$\mathrm{Hr}$ - Hermano
$\mathrm{N}$ - Nieto
$\mathrm{NHr}$ - Nieto de hermano

(1), (2) - Número ordinal de mandato. 


\section{BIBLIOGRAFÍA}

Acerca del trabajo y los trabajadores en el Islam medieval trata la monografía de Maya Shatzmiller Labour in the medieval Islamic World, Leiden, 1994 (sobre la construcción, véanse sobre todo las páginas 209 y siguientes). que contiene bibliografia actualizada. Como introducción especifica a la construcción y los constructores en el mismo ámbito es sumamente útil el capitulo de R. Lewcock "Architects, Craftsmen and Builders: Materials and Techniques": contenido entre las páginas 112-43 de la obra dirigida por $\mathrm{G}$. Michell. Architecture of the /slamic World. Its History and Social Meaning, $1^{\mathrm{a}}$ ed., Londres, 1978. Hay reediciones posteriores - no revisadas ni ampliadas- en inglés y castellano. También contiene bibliografía de referencia. Sobre al-Andalus omeya en particular es básico el artículo de M. Ocaña Jiménez. "Arquitectos y mano de obra en la construcción de la gran mezquita de Occidente". Cuadernos de la Alhambra. 22. 1986, pp. 55-85. pues si bien se centra en un asunto muy concreto - las marcas de identidad en la mezquita aljama de Córdoba_-, traza una excelente panorámica a modo de marco.

Numerosas obras edilicias andalusies entre los siglos vil y $x$ están reseñadas por $L$. Torres Balbás, "Arte hispanomusulmán hasta la caída del califato de Córdoba", en el volumen $\checkmark$ de la Historia de España dirigida por R. Menéndez Pidal, reed. . Madrid, 1982, pp. 331-788. El insigne arquitecto utilizó todos los datos disponibles en su momento. Su trabajo sigue siendo fundamental.

A lo largo de los últimos años hemos tratado de sistematizar noticias procedentes de fuentes escritas y que se refieren a construcciones. Resultados preliminares de nuestra labor pueden verse en "Building (in) Umayyad al-Andalus: remarks in the light of certain written sources", de próxima aparición en la revista Al-Masaq.

Buena parte de la bibliografía sobre inscripciones constructivas omeyas aridalusies la hemos incluido en "Epigraphy and building in Umayyad al-Andalus: genesis and prospects for a research project". Proceedings of the XVIII Congress of the UEAI, en prensa. Sobre las de Madinat az-Zahra', v. M" A. Martinez Núñez, "La epigrafía del salón de 'Abd al-Rahman III", en A. Vallejo Triano, coord., Madinat al-Zahra'. El salón de 'Abd al-Rahman III. Córdoba. 1995. pp. 107-52. Consideraciones parciales acerca de algunas de ellas en A. Fernández-Puertas. "Introducción» a la monografia de Purificación Marinetto Sánchez, Los capiteles del palacio de los Leones en la Altambra. Ejemplo para el estudio del capitel hispanomusulmán y su trascendencia arquitectónica. Estudio I. Granada, 1996. pp. XIX-LIX. Sobre esta ciudad-palacio, véanse también los sucesivos números de la revista Cuadernos de Madinat al-Zahra: Es muy elocuente el título de la contribución de Ana Labarta y Carmen Barceló en el primero de ellos (1987): "Las fuentes árabes sobre al-Zahra": estado de la cuestión". 